\title{
Arbitrable Blind Quantum Computation
}

\author{
Go Sato, ${ }^{1}$ Takeshi Koshiba, ${ }^{2}$ and Tomoyuki Morimae ${ }^{3}$ \\ ${ }^{1}$ Division of Mathematics Electronics and Informatics, \\ Graduate School of Science and Engineering, Saitama University, \\ 255 Shimo-Okubo, Sakura-ku, Saitama 338-8570, Japan \\ ${ }^{2}$ Faculty of Education and Integrated Arts and Sciences, \\ Waseda University, 1-6-1 Nishiwaseda, Shinjuku-ku, Tokyo 169-8050, Japan \\ ${ }^{3}$ Department of Computer Science, Gunma University, \\ 1-5-1 Tenjin-cho, Kiryu-shi, Gunma 376-0052, Japan
}

\begin{abstract}
Blind quantum computation is a two-party protocol which involves a server Bob who has rich quantum computational resource and provides quantum computation service and a client Alice who wants to delegate her quantum computation to Bob without revealing her quantum algorithms and her input to (resp., output from) the algorithms. Since Bob may be truant and pretend to execute some computation, Alice wants to verify Bob's computation. Verifiable blind quantum computation enables Alice to check whether Bob is cheating or not. If Bob is cheating and claims his innocence, Alice can refute the denial of Bob's cheating but she cannot persuade any others that Bob is cheating. In this paper, we incorporate arbitrators as the third party into blind quantum computation to resolve the above problem and give an arbitrable blind quantum computation scheme.

PACS numbers: 03.67.Ac, 03.67.Dd
\end{abstract}

\section{INTRODUCTION}

Secure computation involves several parties who want to evaluate functions over their private inputs without compromising the privacy. Since the notion of secure computation was invented by Yao, there have been many proposals to implement secure computation. The first scheme by Yao [1] was realized as a combination of encrypted circuits (garbled circuits) to evaluate a function and oblivious transfer protocols and its security relied on an unproven computational complexity theoretic assumption. Unfortunately, unconditionally secure computation for general functionalities is impossible in the classical setting [2] and even in the quantum setting 3]. Thus, we have to consider special cases where unconditionally secure computation can be realized. As a special case of secure computation, a protocol (BFK09 protocol) for unconditionally secure delegated computation was shown by Broadbent, Fitzsimons and Kashefi [4]. Secure delegated computation in the quantum setting (a.k.a. blind quantum computation (BQC)) is a two-party protocol between a client (Alice) and a server (Bob): Bob is capable of carrying out any quantum computation and Alice wants to delegate her computational tasks to Bob without revealing her secret, that is, her algorithms and their inputs/outputs. After Broadbent et al's seminal work, BQC protocols in different settings and model have been proposed [5 14].

While BQC protocols ensure Alice's privacy, Alice may not detect Bob's cheating behaviors if Bob neglects Alice's request and pretends to execute her algorithm. To overcome this problem, some BQC protocols support the verifiability which enables to detect Bob's dishonest behavior if Bob is cheating. The Fitzsimons-Kashefi protocol (FK12 protocol) 15] is a verifiable variant of the BFK09 one and the Hayashi-Morimae protocol (HM15 protocol) [16] is a verifiable variant of the Morimae-Fujii BQC one (MF13 protocol) [5]. If Alice can verify Bob's execution of Alice's algorithm, there still exists a problem. Even if Alice finds Bob's cheating behavior, Bob may insist that he is innocent and that Alice rather tries to ensnare him. For example, Alice pays a service fee to Bob if Bob honestly provides the delegated computation service. On the other hand, Alice does not want to pay anything if Bob is cheating. The cheating Bob may charge Alice for his cheating service. Any person outside the protocol (e.g. the FK12 protocol or the HM15 protocol) cannot decide which one is honest.

The notion of verifiability given in [15] is what we should call it private verifiability. We have observed that the private verifiability does not resolve the above dispute. To resolve this dispute problem, we rather need a mechanism where any public outsider or the specific third-party can verify if Bob honestly provides a dele- 
gated computation service. In [17], Honda proposes the notion of public verifiability for BQC and provides a publicly verifiable BQC protocol by using a classical computational cryptography, which relies on an unproven computational assumption. In this paper, we take a different approach from [17] and provide a protocol which does not rely on computational assumptions. To do this, we incorporate arbitrators as the third party into the HM15 protocol and give an unconditionally-secure arbitrable blind quantum computation protocol.

For BQC, there is another approach by using homomorphic encryptions. In the classical setting, Gentry devised a fully homomorphic encryption [18]. Even in the quantum settings, some possibilities of homomorphic encryptions have been discussed [19, 20].

\section{PRELIMINARIES}

As mentioned, we will give an arbitrable blind quantum computation protocol. First, we briefly review the MF13 protocol on which we base our protocol. In our protocol, we need a procedure (i.e., honesty test) to decide if a given state is actually a graph state. For such an honesty test, we may use the Morimae-Nagaj-Schuch test (MNS16 test) 21] or tests in [16, 22, 23] and the security can be proved by using quantum de Finetti Theorem under measurements that are implementable by fully-one-way local operations and classical communication (LOCC) 24].

\section{A. MF13 and HM15 protocols}

A client Alice has only a measurement device and a server Bob prepares a resource state of measurementbased quantum computation. For the MH13 protocol, any resource state (e.g., two-dimensional cluster state or three-dimensional cluster state for topological quantum computation) can be used. The MH13 protocol is as follows: (1) Bob prepares a universal resource state; (2) Bob sends a particle of the resource state to Alice via the quantum channel; (3) Alice measures the particle with respect to the basis which is determined by her algorithm. They repeat (2) and (3) until the computation halts.

The HM15 protocol is a privately-verifiable version of the MF13 protocol. In the HM15 protocol, Bob first prepares $k+1$ copies of the resource state (represented as a bipartite graph). $k$ out of $k+1$ copies are used for stabilizer tests for the verifiability. The remaining one copy is used for the computation where Alice's algorithm is performed via the MF13 protocol.

\section{B. Honesty Test}

Morimae, Nagaj and Schuch 21] gave a verification procedure (MNS16 test) that checks whether a given quantum state $|\psi\rangle$ is close to a graph state $|G\rangle$. The probability the MNS16 test passes is described as $(1+$ $\langle\psi \mid G\rangle) / 2$. While the honesty test in [16] is for bipartitegraph states, the MNS16 test and also tests in 22, 23. are for any graph states. In the protocol description we will show, we mention the MNS16 test for the honesty test. Alternatively, we may use other tests in [16, 22, 23].

\section{PROTOCOL}

As in the standard BQC protocols, we assume that $\mathrm{Al}-$ ice, a client, would like to securely delegate her computation to Bob, a server. We assume that Bob can prepare a universal $n$-qubit graph state $|G\rangle$. Besides Alice and Bob, we assume that there exists a trusted third-party Charlie who acts as an arbitrator. In this paper, we assume that Charlie always obeys the protocol and stands neutral. Our protocol is given in Protocol 1.

For the protocol, we assume that $k \geq 4 n^{2}-1$ and $m \geq(2 \ln 2) k^{n} n^{5}$. It is not essential that Charlie has quantum memory in the protocol. If Charlie does not have quantum memory, he can take the option in STEP 3. Moreover, if Alice considers that her private verification suffices, Charlie does not have to be involved in the protocol. In that case, Bob directly sends $k+m+1$ graph states to Alice in STEP 1; Alice applies a random permutation to $k+m+1$ graph states and discards $m$ graph states in STEP 2; and STEP 3 and STEP 6 can be omitted.

Then, Protocol 1 satisfies the following two properties. (Completeness)

If Bob sends $|G\rangle^{\otimes 2 k+m+1}$ to Alice (via Charlie), then Alice passes the test with probability 1 .

(Soundness)

If Alice passes the test, $\rho_{\text {comp }}$ satisfies $\left\langle G\left|\rho_{\text {comp }}\right| G\right\rangle \geq$ $1-\frac{1}{n}$ with probability $1-\frac{1}{n}$ at least.

The completeness just comes from the construction of Protocol 1. The soundness can be similarly discussed as in [22]. For self-containment, we review a proof in 


\section{Protocol 1 Arbitrable BQC protocol}

STEP 1: Bob generates $|G\rangle^{\otimes 2 k+m+1}$ and sends them to Charlie, where $|G\rangle$ is an $n$-qubit graph state.

STEP 2: Charlie applies a random permutation to $2 k+m+1$ graph states and discards $m$ graph states.

STEP 3: Charlie keeps $k$ graph states in his memory and sends the remaining $k+1$ graph states $\rho$ to Alice. (Optionally, Charlie may execute $k$ MNS16 tests as in STEP 6. If the $k$ tests are not passed, Charlie judges that Bob is cheating.)

STEP 4: Alice receives a graph states $\rho$ and applies the MNS16 tests to randomly chosen $k$ graph states from $k+1$ graph states $\rho$. Let $\rho_{\text {comp }}$ be the remaining one graph state. Alice executes the algorithm on $\rho_{\text {comp }}$ by measuring the particle with respect to the basis which is determined by the description of Alice's algorithm.

STEP 5: If those $k$ tests pass, then Alice accepts the computation. Otherwise, Alice rejects the computation. Charlie does not anything if Alice accepts.

STEP 6: If Alice rejects, then Charlie executes $k$ MNS16 tests by using $k$ graph states stored in his memory. If those $k$ tests are passed, Charlie judges that Alice is cheating. Otherwise, Charlie judges that Bob is cheating.

[1] A. C.-C. Yao, Proc. 27th Ann.Symp. Found. Comp. Sci., 162 (IEEE Computer Society, 1986).

[2] R. Cleve, Proc. 18th Ann. Symp. Theor. Comp., 364 (ACM, 1986).

[3] H.-K. Lo, Phys. Rev. A 56, 1154 (1997).

[4] A. Broadbent, J. Fitzsimons and E. Kashefi: Proc. 50th Ann. Symp. Found. Comp. Sci., 517 (IEEE Computer Society, 2009).

[5] T. Morimae and K. Fujii, Phys. Rev. A 87, 050301(R) (2013).

[6] T. Sueki, T. Koshiba, and T. Morimae, Phys. Rev. A 87, 060301(R) (2013).

[7] T. Morimae and K. Fujii, Nat. Comm. 3, 1036 (2012).

[8] T. Morimae, Phys. Rev. Lett. 109, 230502 (2012).

[9] V. Dunjko, E. Kashefi, and A. Leverrier, Phys. Rev. Lett. 108, 200502 (2012).

[10] V. Giovannetti, L. Maccone, T. Morimae, and T. G. Rudolph, Phys. Rev. Lett. 111, 230501 (2013).

[11] A. Mantri, C. A. Perez-Delgado, and J. F. Fitzsimons, Phys. Rev. Lett. 111, 230502 (2013).

[12] T. Morimae and K. Fujii, Phys. Rev. Lett. 111, 020502 (2013).

[13] V. Dunjko, J. F. Fitzsimons, C. Portmann, and R. Renner, Lect. Notes Comp. Sci. 8874, 406 (2014).

[14] T. Morimae, V. Dunjko, and E. Kashefi, Quant. Inf. Comput. 15, 0200 (2015).
Appendix.

On Eq.A.1 in Appendix, we suppose that $T$ is a POVM for Alice in STEP 5. On the other hand, we can provide another interpretation for Eq.A.1 and consider that $T$ is a POVM for Charlie in STEP 6. Even in this interpretation, we have a similar consequence. That is, if Charlie passes the test, $\rho_{\text {comp }}$ satisfies $\left\langle G\left|\rho_{\text {comp }}\right| G\right\rangle \geq$ $1-\frac{1}{n}$ with probability $1-\frac{1}{n}$ at least. In other words, if Alice accepts the computation, then Charlie can endorse her acceptance. Thus, we can say that Charlie works as an arbitrator.

\section{Acknowledgments}

TK is supported in part by JSPS Grant-in-Aids for Scientific Research (A) 16H01705 and for Scientific Research (B) 17H01695. TM is supported by JST ACT-I No.JPMJPR16UP and a JSPS Grant-in-Aid for Young Scientists (B) $17 \mathrm{~K} 12637$.

[15] J. Fitzsimons and E. Kashefi, arXiv:1203.5217 (2012).

[16] M. Hayashi and T. Morimae, Phys. Rev. Lett. 115, 220502 (2015).

[17] K. Honda, arXiv:1604.00116 (2016).

[18] C. Gentry, Proc. 41st Ann. Symp. Theor. Comp., 169 (ACM, 2009).

[19] A. Broadbent and S. Jeffery, Lect. Notes. Comp. Sci. 9216, 609 (2015).

[20] Y. Dulek, C. Schaffner, and F. Speelman, Lect. Notes Comp. Sci. 9816, 3 (2016).

[21] T. Morimae, D. Nagaj, and N. Schuch, Phys. Rev. A 93, 022326 (2016).

[22] T. Morimae, Y. Takeuchi, and M. Hayashi, arXiv:1701.05688 (2017).

[23] Y. Takeuchi and T. Morimae, arXiv:1709.07575 (2017).

[24] K. Li and G. Smith, Phys. Rev. Lett. 114, 160503 (2015).

\section{Appendix: Proof of the Soundness Property}

First, for any $n$-qubit quantum state $\sigma$, we can show that

$$
\operatorname{Tr}\left[\left(T^{\otimes k} \otimes \Pi_{G}^{\perp}\right) \sigma^{\otimes k+1}\right] \leq \frac{1}{2 n^{2}},
$$


where $T$ is a POVM which corresponds to a pass by the honesty test and

$$
\Pi_{G}^{\perp}=I^{\otimes n}-|G\rangle\langle G| .
$$

Since

$$
\operatorname{Tr}(T \sigma)=\frac{1}{2}+\frac{1}{2}\langle G|\sigma| G\rangle
$$

we can say that

$$
\begin{aligned}
\operatorname{Tr}\left(\Pi_{G}^{\perp} \sigma\right) & =1-\langle G|\sigma| G\rangle \\
& =2(1-\operatorname{Tr}(T \sigma)) .
\end{aligned}
$$

Thus, we have

$$
\begin{aligned}
\operatorname{Tr}\left[\left(T^{\otimes k} \otimes \Pi_{G}^{\perp}\right) \sigma^{\otimes k+1}\right] & =\operatorname{Tr}(T \sigma)^{k} \operatorname{Tr}\left(\Pi_{G}^{\perp} \sigma\right) \\
& =2 \operatorname{Tr}(T \sigma)^{k}(1-\operatorname{Tr}(T \sigma)) .
\end{aligned}
$$

When $\operatorname{Tr}(T \sigma)=\frac{k}{k+1}$, the above takes the maximum value

$$
2\left(\frac{k}{k+1}\right)^{k}\left(1-\frac{k}{k+1}\right) \leq \frac{2}{k+1} \leq \frac{1}{2 n^{2}}
$$

The remaining $(k+1)$ qubits quantum state $\rho$ after the trace-out can be obtained as follows by using the quantum de Finetti theorem with respect to the one-way LOCC norm

$$
\begin{aligned}
& \operatorname{Tr}\left[\left(T^{\otimes k} \otimes \Pi_{G}^{\perp}\right) \rho\right] \\
& \leq \int d \mu(\sigma) \operatorname{Tr}\left[\left(T^{\otimes k} \otimes \Pi_{G}^{\perp}\right) \sigma^{\otimes k+1}\right]+\frac{1}{2} \sqrt{\frac{2 k^{2} n \ln 2}{m}} \\
& \leq \frac{1}{2 n^{2}}+\frac{1}{2 n^{2}}=\frac{1}{n^{2}} .
\end{aligned}
$$

Since

$$
\operatorname{Tr}\left[\left(T^{\otimes k} \otimes \Pi_{G}^{\perp}\right) \rho\right]=\operatorname{Tr}\left(\Pi_{G}^{\perp} \rho_{\text {comp }}\right) \operatorname{Tr}\left(T^{\otimes k} \rho\right),
$$

$\operatorname{Tr}\left(\Pi_{G}^{\perp} \rho_{\text {comp }}\right) \geq \frac{1}{n}$ implies that $\operatorname{Tr}\left(T^{\otimes k} \rho\right) \leq \frac{1}{n}$. This means that If Alice accpets, then it holds that $\left\langle G\left|\rho_{\text {comp }}\right| G\right\rangle \geq 1-\frac{1}{n}$ with probability $1-\frac{1}{n}$ at least. Thus the soundness holds. 